\title{
Frequency, Distribution and Genotyping of Malassezia Species in Patients with Psoriasis vulgaris
}

\author{
Ebru Celik ${ }^{1}$, Nizami Duran², Didem Didar Balci ${ }^{3}$, Asena Cigdem Dogramaci ${ }^{1}$, \\ Ozgur Pasa ${ }^{4}$ \\ 1. Department of Dermatology, Medical Faculty, Hatay Mustafa Kemal University, Turkey \\ 2. Department of Microbiology, Medical Faculty, Hatay Mustafa Kemal University, Turkey \\ 3. Department of Dermatology, Izmir Tepecik Training and Research Hospital, Turkey \\ 4. Department of Medical Microbiology, Tatvan State Hospital, Turkey
}

\begin{abstract}
Background: Malassezia species are reported to play a role in the etiology of Psoriasis vulgaris. Objective: The aim of this study was to determine the presence, frequency, distribution, and genotyping of skin colonization of Malassezia species in Psoriasis vulgaris and to compare with healthy individuals and to investigate its relationship with the severity of the disease. Methods: Skin samples were taken from scalp, arm, body, and leg of 34 psoriasis patients (lesional/ non-lesional skin) and 30 healthy volunteers. Overall, 392 skin scraping samples were taken for the isolation of Malassezia species, which were incubated on the modified-Dixon agar. Conventional culture methods were used for Malassezia species identification. In isolates, genotyping was carried out by PCR-RFLP method. Results: In the samples from psoriatic lesions, most frequently isolated Malassezia species were M.globosa and M.furfur. Similarly, the most frequently isolated species in healthy volunteers was M. globosa; followed by M.restricta and M.sympodialis. The M.furfur isolation rate in psoriatic scalp and leg lesions of the patients was significantly higher than in healthy volunteers. There was no relationship between the severity of the disease and the isolated species. Conclusion: It was found that there was a difference between patients with psoriasis and healthy controls regarding presence and frequency of Malassezia species. Therefore, our study results support the view that Malessezia species may be associated with the etiopathogenesis of psoriasis. In addition, we surmise that the treatment applications for the regulation of skin microbiota of psoriasis patients will contribute positively to the treatment of psoriasis.
\end{abstract}

Keywords: psoriasis vulgaris, malassezia, PCR-RFLP, genotyping, modified-Dixon agar, microbiota Received: 20 th August 2020; Accepted: 14 ${ }^{\text {th }}$ January 2021; Published: $24^{\text {th }}$ February 2021

\footnotetext{
* Corresponding author: Nizami Duran, Microbiology, Hatay Mustafa Kemal University, Medical Faculty, Turkey E-mail: nduran@mku.edu.tr
} 


\section{Introduction}

Psoriasis vulgaris with unkown etiology is a chronic, recurrent, immune-mediated, inflammatory skin disease which is common in the society. It is thought that the disease is associated with hereditary polygenic predisposition as well as the irregular interaction between environmental triggers, including keratinocytes, immune cells, and microbiota $(1,2)$. In recent years, it has been reported that changes in skin microbiota (dysbiosis) play a role in the pathogenesis of chronic plaque psoriasis disease. It is reported that some skin microbiota members, such as Streptococcus, Staphylococcus, and Cutibacterium, as well as Malassezia and Candida may aggravate psoriasis. It is also suggested that the cellular immune response thought to be developing against these agents may be responsible for the pathogenesis (3-6). It has been reported that lesions compatible with psoriasis occur after the application of inactivated Malassezia species to the non-lesional skin of patients with psoriasis by patch test method (7). In addition, since the soluble components obtained from Malassezia are chemoattractant for polymorphonuclear leukocytes of psoriatic patients, it has been shown that Malassezia species play a role in koebnerization of psoriasis (8-9).

Malassezia species are among the fungal members of commensal skin microbiota. These species have been reported to be associated with some skin diseases. Fourteen species of Malassezia fungi are known today. Some of them are zoophilic and some of them are located in humans as members of human microbiota including M.furfur, M.sympodialis, M.globosa, M.obtusa, M. restricta, M.slooffiae, M.dermatis, M.japonica, M.yamatoensis (10). Malassezias prefer fat-rich body areas in humans, and their density varies in the area according to localization (11). They are especially colonized particularly in the scalp, upper body, and flexural areas.
Malassezia species do not infect hair, nails, and mucous membranes (12).

There are controversial results in the studies on the clinical significance of Malassezia species since in vitro cultivation is difficult for these organisms and there are challenges in their identification (13). Special media are needed for the growth of Malassezia species; thus, limited study has been carried out so far. Modified Dixon agar containing peptone (14) has been reported as the most suitable medium for isolation of Malassezia species (15).

The aim of this study was to determine the presence, frequency, distribution, and genotyping (by PCR-RFLP method) of Malassezia species in samples taken from patients with Psoriasis vulgaris and to compare with healthy individuals. In addition, we also investigated whether there is a relationship between Malassezia species and Psoriasis area severity index (PASI).

\section{Materials and Methods}

The study was approved by the Ethics Committee of Hatay Mustafa Kemal University, Faculty of Medicine (2009-47/ 05-20). Overall, 64 subjects were included in the study: 34 patients diagnosed with psoriasis and 30 healthy individuals who presented to the Dermatology Outpatient Clinic of Hatay Mustafa Kemal University Faculty of Medicine between 2010 and 2011.

All subjects signed and informed consent before participation. In the study, demographic data (age, gender, and disease duration of the patients) of the individuals included were collected by face-to-face interview method. In addition, PASI values were calculated and recorded in all patients. In the study, exclusion criteria comprised: the use of systemic steroid and other immunosuppressive therapy, and systemic or topical antifungal therapy within the prior month, topical steroid use within the prior week, and moisturizer use within the prior 24 hours, bathing within 
the prior 3 days and the presence of immunosuppressive disease, disability or pregnancy.

\section{Mycological evaluation}

In the study, both psoriatic lesional skin samples (136 pieces) and non-lesional skin samples (136 pieces) were taken from the patients. Samples from four different body regions (scalp, arm, body, and leg) were taken from healthy volunteers. Overall, 120 skin scraping samples were taken from healthy volunteers. Before taking the samples, the skin area was cleaned using $70 \%$ ethyl alcohol and the samples were taken with sterile scalpel after waiting for a while. Overall, 392 specimens taken from the skin as scrapings were inoculated to modified Dixon agar (mDIX; Oxoid, UK). Each sample was inoculated on two petri plates. The inoculated plates were incubated for 10 days at $32^{\circ} \mathrm{C}$ under aerobic conditions. In all plates, growth was controlled daily. At the end of incubation, microorganisms with in yeaststyle growth were tested with conventional techniques to determine whether they were Malassezia (16).

\section{Isolation of Malassezia Species}

Clinical samples were taken under sterile conditions. The isolation of the samples was carried out according to the method described by Bayraktar et al. (17). After the samples were inoculated into Dixon agar plates, sterile olive oil was added to cover the surface of the medium. Plates were incubated at aerobic conditions for 10 days at $32^{\circ} \mathrm{C}$.

\section{Polymerase chain reaction (PCR)}

Genomic DNA extraction was performed using commercially available glass bead isolation kits (Roche, ZR Fungal DNA Kit, Germany). DNA isolation was performed in accordance with the test procedure. The following primary sequence based on the 26S rDNA sequence of Malassezia species was used for PCR amplification (forward: 5'-TAACAAGGATTCCCCTAGTA 3', reverse: 5'ATTACGCCAGCATCCTAAG 3'). PCR amplification was prepared as follows: with the final volume of $50 \mu \mathrm{L} .1 \mu \mathrm{L}$ template DNA, $0.5 \mu \mathrm{M}$ from each primer, $0.20 \mathrm{mM}$ from each deoxynucleotide triphosphate (dNTPs; AB gene, UK), $5 \mu \mathrm{L} \mathrm{10x}$ PCR buffer ( $\mathrm{MgCl} 2$ Chlorine-free; Promega corp.) and 1.25 U Taq DNA polymerase (Fermentas, USA).

PCR amplification was performed in the Thermal cycler (Bioder/ Thermal Blocks xp cycler, Tokyo Japan) in the following steps. The first denaturation; 5 minutes at $94^{\circ} \mathrm{C}$, denaturation; 45 seconds at $94^{\circ} \mathrm{C}$, annealing; 45 seconds at $55^{\circ} \mathrm{C}$, 1 minute at elongation at $72^{\circ} \mathrm{C}, 7$ minutes at last at $72^{\circ} \mathrm{C}$. Denaturation, annealing, and elongation periods were applied for 30 cycles.

The amplified PCR products were digested with CfoI (Roche Diagnostics, Mannheim Germany) and BstF51 (Sib Enzyme, Russia) restriction endonuclease enzymes. Genotyping of Malassezia species (Malassezia (M).globosa, M.furfur, M.restricta, M.sympodialis, M.slooffiae, M.obtusa and Malassezia spp.) was performed according to species-specific band patterns. The final volume was adjusted to $25 \mathrm{ml}$ for the restriction procedure. For digestion, PCR-amplified DNA and the restriction enzymes were incubated for 3 hours at $37^{\circ} \mathrm{C}$. Thereafter, the presence of specific amplification products for Malassezia species was visualized by running on a $1.5 \%$ agarose gel (Wealtec, Dolphin- View, USA).

\section{Statistical Analysis}

Categorical measurements are summarized as number and percentage, while numerical measurements are summarized as average and standard deviation. Chi-Square test was used to analyzenon-parametric data in the patient and the healthy control group. Mc Nemar test was used to compare patients regarding Malassezia species that were determined from lesional and non-lesional skins. Mann-Whitney U test was 
used to determine the relation between Malassezia species and PASI. In addition, Spearman correlation test was performed to compare the disease duration of psoriatic patients with presence of Malassezia species. Statistical significance level was taken as $p<0.05$ in all tests. SPSS 16.0 package software was used in the statistical analysis of data.

\section{Results}

The study included 34 patients diagnosed with Psoriasis vulgaris (18 men, 16 women) and 30 healthy volunteers (14 men, 16 women). The mean ages of the patients and healthy volunteers were $42.0 \pm 13.3$ and $41.7 \pm 11.9$ years, respectively. The average disease duration was $12.1 \pm$ 8.6 years. Mean PASI values were determined as $12.7 \pm 8.9$ in the patients.

The culture positivity rate was determined as $55.9 \%(76 / 136)$ in the samples taken from psoriatic lesions and $48.5 \%(66 / 136)$ in the samples taken from non-lesional areas in the patient group whereas $32.5 \%(39 / 120)$ in healthy volunteers. There was no statistically significant difference in culture positivity between the lesional and non-lesional skin areas of patients with psoriasis $(p=0.203)$. Statistically significant difference was found in culture positivity between the samples from the lesional areas of patients with psoriasis and the samples from healthy volunteers $(p<0.001)$. Similarly, a statistically significant difference was found between non-lesional skin samples of patients and samples taken from volunteers $(p=0.011)$.

\section{PCR results}

The presence of Malassezia species, i.e. PCR positivity, isolated and confirmed by PCR was compared with patients with psoriasis and healthy volunteers. The PCR positivity rate of Malassezia species was found to be significantly higher in the patients from a statistical point of view.
PCR positivity was found in $59.6 \%(81 / 136)$ of the samples taken from the lesions of the patients with psoriasis and $51.5 \%(70 / 136)$ of the samples taken from the non-lesional skin. In healthy volunteers, it was found as $34.2 \%(41 / 120)$. In samples taken from psoriatic-lesional and non-lesional areas of patients with psoriasis, there was no statistically significant difference in terms of PCR positivity ( $p=0.127$ ). However, PCR positivity in samples taken from psoriatic lesions was found to be significantly higher than healthy volunteers $(p<0.001)$. Similarly, a statistically significant difference was found between the samples taken from the lesions-free areas of the patients and the volunteers $(p=0.008)$.

PCR positivity of Malassezia species in psoriatic-lesional and non-lesional samples according to anatomic regions in psoriasis patients were as follows: $61.8 \%$ and $50.0 \%$ on the scalp, $61.8 \%$ and $44.1 \%$ on the arm, $58.8 \%$ and $55.9 \%$ on the body, $55.9 \%$ and $55.9 \%$ on the leg, respective1y. No statistically significant difference was detected in PCR positivity between lesional and non-lesional skin areas $(p>0.05)$. PCR positivity was found as $56.7 \%, 26.7 \%, 40.0 \%$, and $13.3 \%$ on the scalp, arm, body, and leg samples of healthy volunteers, respectively.

The most common Malassezia species isolated in the psoriatic lesional and non-lesional skin samples of psoriasis patients were M.globosa and M.furfur. The rate of M.globosa was determined as $30.1 \%(41 / 136)$ in psoriatic lesional skin and $24.3 \%(33 / 136)$ in non-lesional skin. The rate of M. furfur was detected as $23.5 \%(32 / 136)$ in psoritic lesional skin and $18.4 \%(25 / 136)$ in non-lesional skin. Malassezia species isolated from the skin samples of patients and healthy volunteers were compared. The frequency of M.furfur was found to be significantly higher in the lesional scalp $(p=0.015)$ and leg $(p=0.029)$ samples of the patients.

The most commonly isolated species in healthy volunteers were found to be M.globosa $21.7 \%$ 
(26/120), M.restricta $13.3 \% \quad(16 / 120)$, and M.sympodialis $11.7 \%(14 / 120)$. In the scalp samples, M.restricta (43.3\%) and M.globosa (23.3\%) were the two most frequently isolated species. In the body, M.globosa (36.7\%), M.sympodialis (20.0\%), and M.slooffiae (13.3\%) were the three most frequently isolated species. Nevertheless, Malassezia colonization in the arms and legs was found to be less than other areas. M.globosa was the most frequently isolated species in the arm $(16.7 \%)$ and leg samples $(10.0 \%)$, followed by M.sympodialis $(13.3 \%-6.7 \%)$. Statistically, when the lesional/non-lesional skin samples of psoriasis patients and healthy volunteers were compared, isolation of M.restricta, M.sympodialis, M.slooffiae (4.2\%) species was significantly higher than the healthy group.

Unidentified Malassezia species, namely Malassezia spp., were found in the lesional skin of patients with psoriasis by $8.8 \%(12 / 136)$ and in non-lesional skin by $11.0 \%(15 / 136)$. The incidence of Malassezia spp. in healthy volunteers was found to be $5.8 \%(7 / 120)$.

In addition, mixed Malessezia colonization was detected in some samples. Psoriasis patients often had M.globosa plus M.furfur, while healthy individuals had M.globosa plus M.sympodialis. The rate of detection of mixt Malassezia colonization in PCR was $11.0 \%(15 / 136)$ in lesions of patients with psoriasis, $6.6 \%(9 / 136)$ in non-lesional skin and $16.7 \%(20 / 120)$ in healthy volunteers.

The skin colonization frequency of the Malassezia species was compared with the PASI value showing the severity of the disease, and no statistically significant difference was found for any Malassezia species $(p>0.05)$. No significant relation was found between age, gender, disease duration, and presence of Malassezia species $(p>0.05)$.

Figure 1 (A-C) presents distribution of Malassezia species isolated from samples taken from different body regions of healthy volunteers, lesional, and non-lesional patient skin. Figure 2 presents comparison of the Malassezia species distributions isolated from psoriatic- lesional and non-lesional samples. Figure 3 presents comparison of the Malassezia species distributions isolated from patients psoriatic lesions and healthy volunteers.

\section{Discussion}

The mechanism of Malassezia in the pathogenesis of psoriasis disease has not been fully elucidated yet. It is suggested that the lipid-rich capsules of Malassezia species mask the recognition of the host defense cells and the reduction of this layer reveals the inflammatory response (18). It is reported that the lipid layer of Malassezia species regulates cytokines produced in keratinocytes. However, Malassezia strains with lipid capsules removed have been reported to significantly increase IL-6, IL-8, IL-1, and TNF- $\alpha$ production and reduce intracellular IL-10 levels $(18,19)$. Malassezia species play an immunomodulatory role with a lipid-rich structure and this feature is important in understanding the ability to cause disease (11). Malassezia species can activate the complement system from the alternative route and the classic route (20). It has been reported that a specific humoral immune response develops against the yeast form of $M$. furfur (formerly known as Pityrosporum ovale) in patients with psoriasis (21). It has been reported that the downregulation effect of Malassezia species on anti-inflammatory cytokines may be related to the appearance of guttate psoriasis (22). In in vitro experiments, it has been reported that the psoriasis patients with M.furfur positivity increase the transforming growth factor-beta 1 , integrin and heat shock protein-70 which lead to psoriasis-like phenotype with keratinocyte migration, hyperproliferation (23). Malassezia species have been shown to play a role in triggering psoriasis by increasing the expression 
A

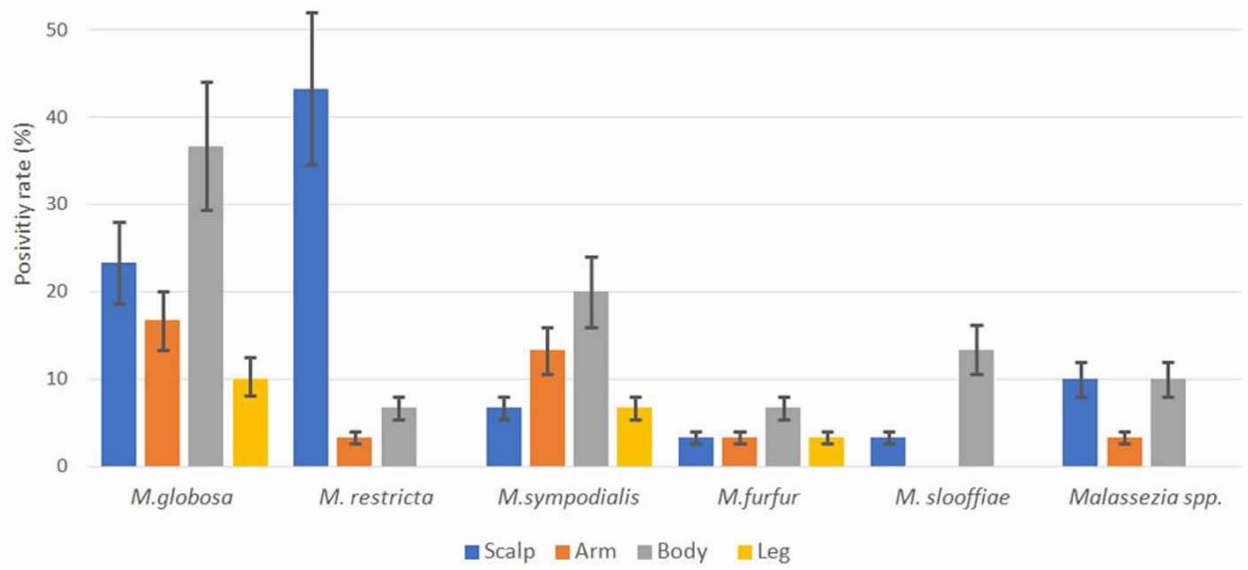

B

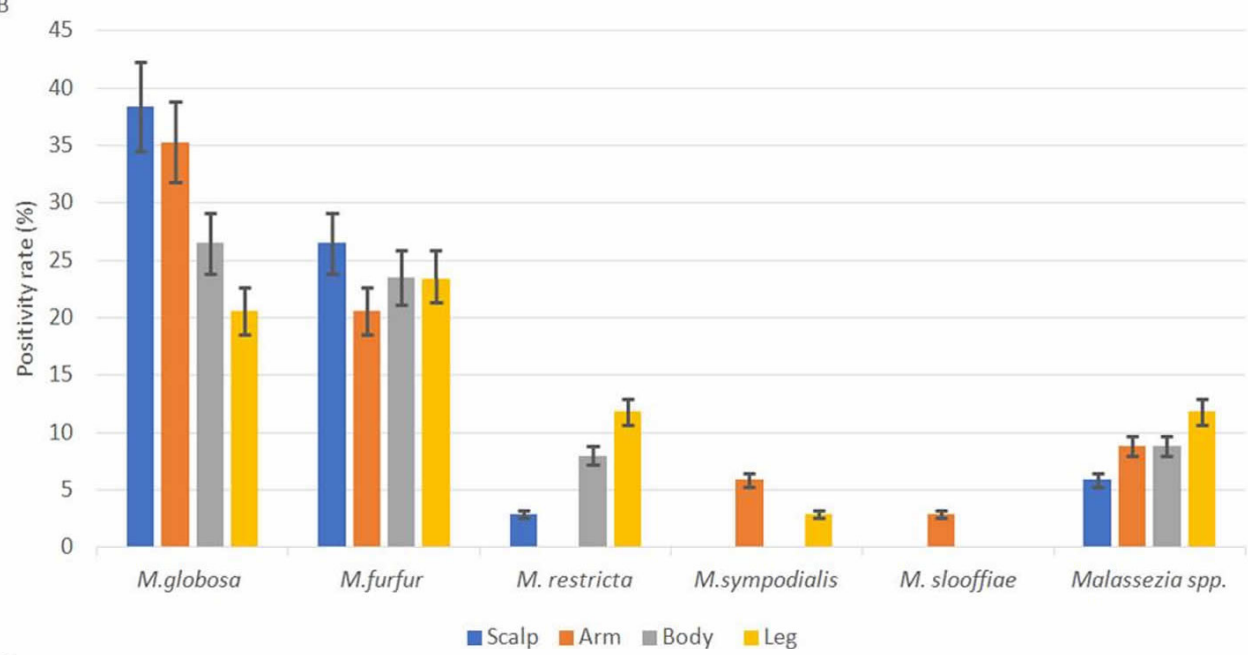

C

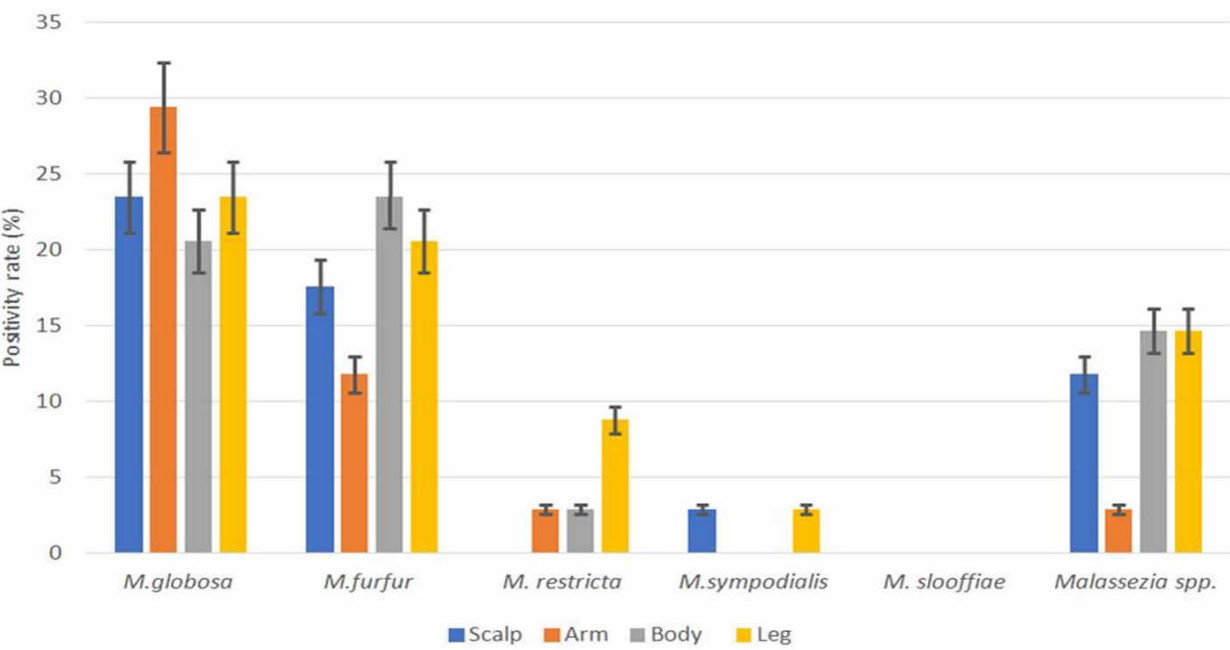

Fig. 1(A-C). Distribution of Malassezia species isolated from samples taken from different body regions of healthy volunteers (A), and psoriatic-lesional (B), non-lesional (C) body regions of psoriasis patients. 


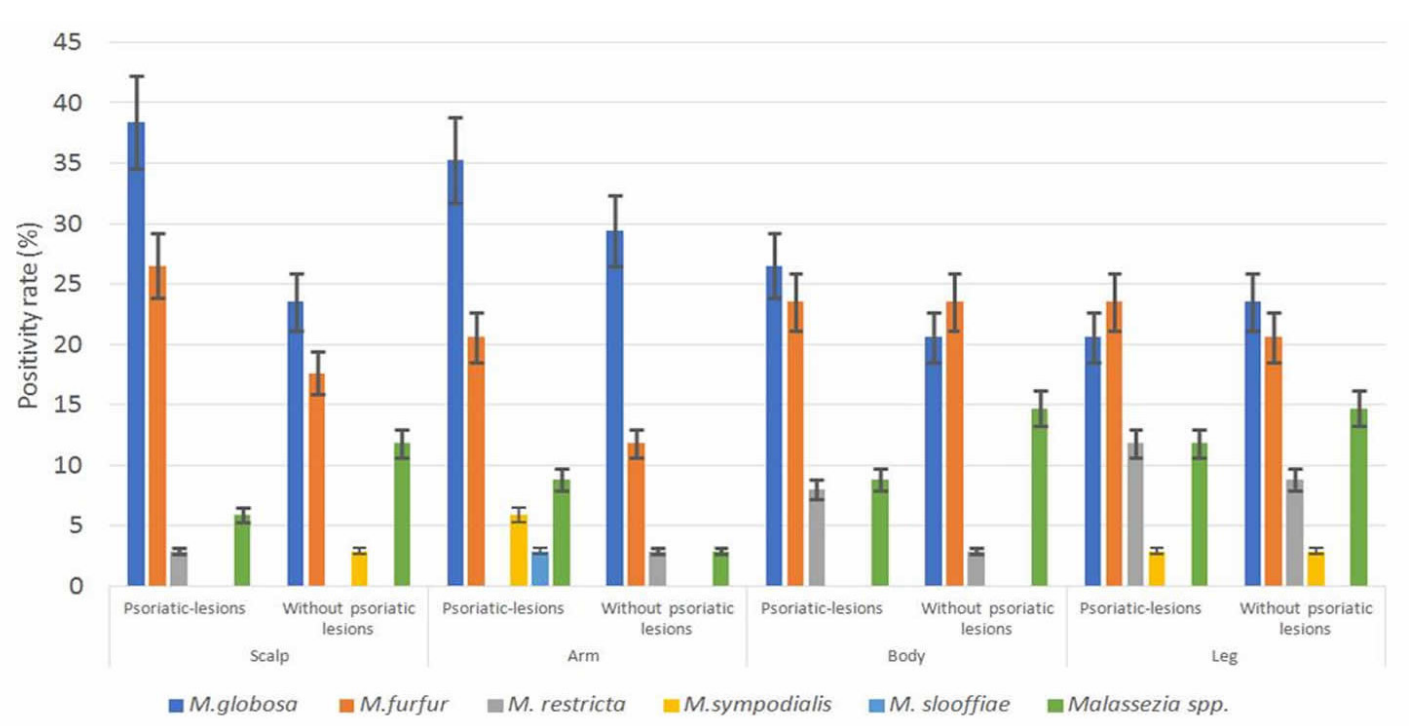

Fig. 2. Comparison of the Malassezia species distribution isolated from psoriatic-lesional and non-lesional skin samples.

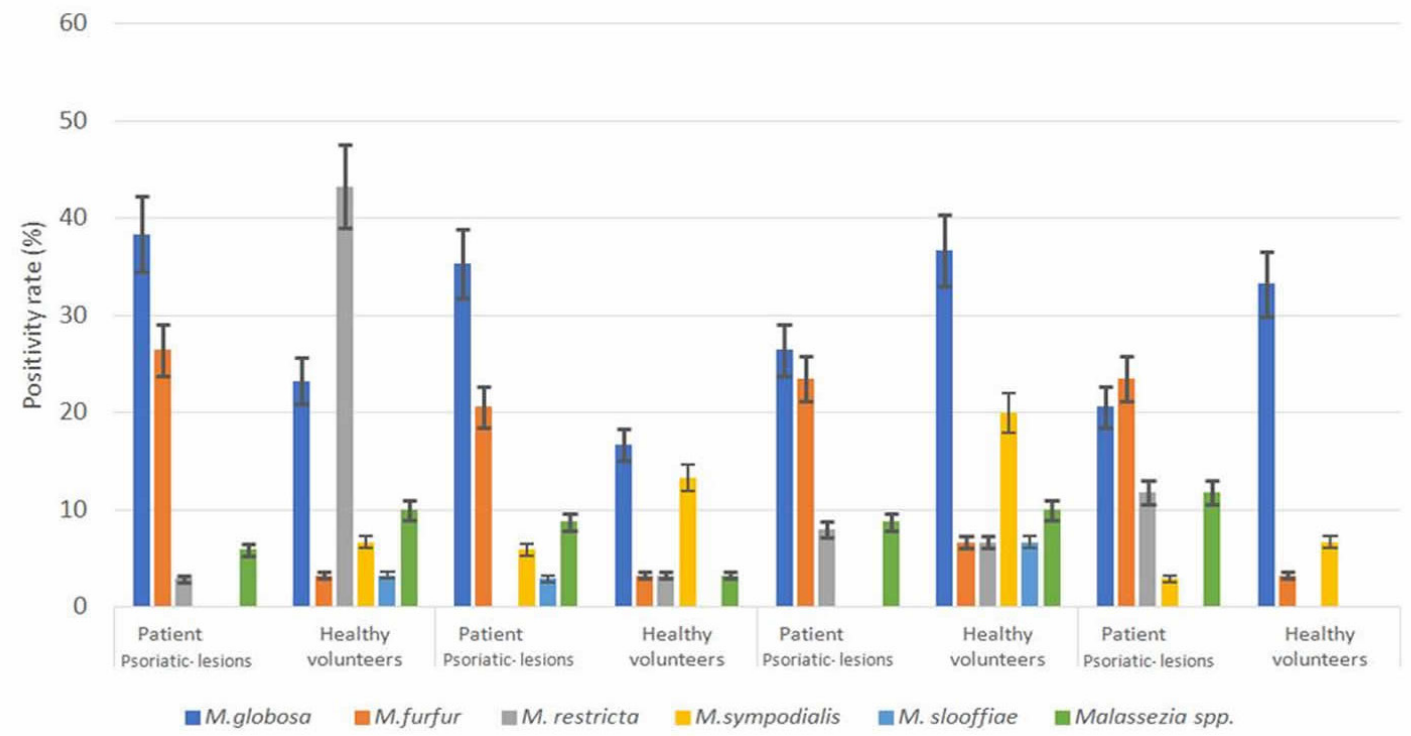

Fig. 3. Comparison of the Malassezia species distribution isolated from patients with psoriatic-lesions and healthy volunteers.

of Toll-like receptor 2, human 32 defensin, and IL-8 (24). In some studies, it has been reported that M.furfur can provoke an exacerbation of psoriatic symptoms. It has been detected that arachidonic acid and metabolites, known as skin inflammation mediators, involved in the hyper- proliferative process of psoriasis are released from epithelial cell membranes by secreting lipase from M.furfur $(5,25,26)$.

The PCR-RFLP technique is successfully used for the identification of Malassezia species in psoriatic skin lesions. It has been reported that 
PCR-RFLP is a more reliable and accurate method in the detection of Malassezia species than Nested-PCR method (27). Therefore, in our study, PCR-RFLP method was used for genotyping of Malassezia species. Zomorodian et al. (5) compared psoriasis patients with healthy individuals in terms of the presence of Malassezia. Skin samples were cultured in modified Dixon agar and identified by PCR-RFLP method. They found that $87 \%$ of healthy individuals and $62 \%$ of psoriasis patients had Malassezia culture positivity. They reported that the isolation rate was higher in the head and extremities in the patient group, and on the head and trunk in healthy individuals. In our study, Malassezia culture positivity was $55.9 \%$ in lesional skin of the psoriasis patient group, $48.5 \%$ in non-lesional skin samples and $32.5 \%$ in healthy volunteers. A statistically significant difference was found between patients with psoriasis and healthy volunteers. In accordance with the literature (5), the positivity of Malassezia was high in lesional scalp and arm in patients with psoriasis in our study. Similarly, positivity was found to be high in the scalp and body in the healthy control group. In our study, there was no statistically significant difference in Malassezia positivity when the skin samples with psoriatic lesions were compared with the non-lesional skin samples in the patient with psoriasis $(\mathrm{p}>0.05)$.

It has been found that M.restricta and M.globosa species are common in healthy individuals in the most of the studies conducted with various methods such as culture, nested-PCR, real time PCR or PCR-RFLP to identify Malassezia species (3, 28-31). In some studies, it has been reported that the most frequently isolated species were M.globosa, but the secondary species M.furfur (5) and M.sympodialis in healthy volunteers (32). On the contrary, some researchers reported that the most isolated species was M.sympodialis $(33,34)$. In our study, in accordance with the literature, $M$. globosa was isolated most frequently in healthy individuals; followed by M.restricta, M.sympodialis, Malassezia spp, M.furfur, and M.slooffiae species. In addition, M.restricta on the scalp of healthy individuals and M.globosa on the body were the most frequently isolated species. The frequency of isolation of Malassezia species on the arm and leg was low. In two regions, M.sympodialis and M.globosa were the most frequently isolated species. However, mixed Malassezia colonization was higher in healthy individuals than in the patient group.

It has been reported in the literature that the most frequently isolated species in the skin samples of patients with psoriasis are M.globosa $(5,22,28,31,33,35)$ and M.restricta $(3,29,30,36,37)$. In some studies, it has been reported that M.furfur is frequently isolated $(34,38)$. It was emphasized that the isolation of different Malassezia species compositions in the current studies in the literature may be related to the difference of the countries in which the studies are conducted $(34,39)$.

A study reported that M.globosa and M.furfur were major species in the lesional skin of the patients with psoriasis (5). Tett et al. (35) emphasized the diversity of skin microbiome in psoriasis patients. They detected that M.globosa and M.restricta were dominant type of fungus. However, they also pointed out the availability of many yet-not-defined genomes named Malassezia spp. In our study, M.globosa, M.furfur, Malassezia spp. (yet-not-defined genomes), M.restricta, M.sympodialis, M.slooffiae were among the isolated Malassezia species. It was found that the species diversity isolated was quite high in accordance with the literature.

Prohic (28) and Gomez-Moyano et al. (31) reported that M.globosa was the most common species in the scalp of patients with psoriasis. Also, they reported that M.restricta was the most common species in the scalp of healthy persons. A study conducted using real-time PCR method by Paulino et al. (30) found that the most fre- 
quently isolated species in both psoriasis patients and healthy individuals were M.restricta and $M$. globosa. It was determined that M.sympodialis was found at a higher rate in the back region of patients with psoriasis than healthy individuals. In another study, the presence of M.restricta in the back region of the patients with psoriasis and M.sympodialis from the elbow area was detected. In healthy individuals, it has been reported that Malassezia spp. and M.slooffiae are highly isolated (37). Zomorodian et al. (5) reported in their study that M.globosa was the dominant species and the second frequent species was M.furfur on all areas (head, trunk, and extremity) psoriatic skin. In our study, M.globosa and M.furfur were found to have highest frequency in lesional and non-lesional skin in the patient group. A significant difference was detected statistically on scalp and leg in terms of lesional area upon comparing the presence of M.furfur in lesional skin with the control group. This confirms the role of M.furfur in psoriasis etiopathogenesis $(5,25,26)$.

In various studies in the literature (36), it was reported that there was no relationship between Malassezia species isolated from patients with psoriasis and PASI score. However, it was reported that there was no relationship between isolated species from patients with psoriasis and the age and gender of the patients. In our study, in accordance with the literature, no relation was found between the PASI score and the Malassezia species isolated from the patients. Similarly, there was no significant relationship between isolated species and age and gender.

The limitation of this study is that a small number of psoriasis patients was included. The strengths of our study include the evaluation of fungal distribution and frequency by taking samples from four different anatomical regions, statistical comparison of the data of the patient group with the data of healthy individuals, the cultivation of Malassezia species in modified Dixon agar, and genotyping using the PCR-RFLP method.
In conclusion, the presence and frequency of Malassezia species in psoriatic patients was found to be higher than healthy volunteers in our study. In addition, the distribution of species isolated from patients with psoriasis has been found to differ from healthy individuals. The fact that Malassezia species isolated from patients with psoriasis are different from healthy individuals and higher isolation rate supports the view that Malassezia species found commensally in the skin microbiome may be related to the etiopathogenesis of psoriasis. In this context, we believe that there is a need to develop prebiotic/ probiotic applications or new treatment strategies such as skin microbiome transfer to correct the current dysbiosis in Psoriasis vulgaris. For this purpose, large-scale studies are needed to develop microflora compatible with skin microbiome of healthy individuals.

\section{Conflict of interest}

The authors declare no conflict of interest.

\section{Acknowledgments}

The study was supported by Hatay Mustafa Kemal University Scientific Research Fund as project number 04M0103/ 2009, Hatay, Turkey.

\section{References}

1. Armstrong AW, Read C. Pathophysiology, clinical presentation, and treatment of psoriasis: A review. JAMA. 2020 May 19;323(19):1945-60. DOI: 10.1001/ jama.2020.4006

2. Lee EB, Wu KK, Lee MP, Bhutani T, Wu JJ. Psoriasis risk factors and triggers. Cutis. 2018 Nov;(5S):18-20.

3. Takemoto A, Cho O, Morohoshi Y, Sugita T, Muto M. Molecular characterization of the skin fungal microbiome in patients with psoriasis. J Dermatol. 2015 Feb;42(2):166-70. DOI: 10.1111/1346-8138.12739

4. Lewis DJ, Chan WH, Hinojosa T, Hsu S, Feldman SR. Mechanisms of microbial pathogenesis and the role of the skin microbiome in psoriasis: A review. Clin Dermatol. 2019 Mar-Apr;37(2):160-6. DOI: 10.1016/j. clindermatol.2019.01.011

5. Zomorodian K, Mirhendi H, Tarazooie B, Zeraati H, 
Hallaji Z, Balighi K. Distribution of Malassezia species in patients with psoriasis and healty individuals in Tehran, Iran. J Cutan Pathol. 2008 Nov; 35811:1027-31. DOI: $10.1111 / \mathrm{j} .1600-0560.2007 .00968 . \mathrm{x}$

6. Benhadou F, Mintoff D, Schnebert B, Thio HB. Psoriasis and microbiota: A systematic review. Diseases. 2018 Jun;6(2):47. DOI: 10.3390/diseases6020047

7. Lober CW, Belew PW, Rosenberg EW, Bale G. Patch tests with killed sonicated microflora in patients with psoriasis. Arch Dermatol. 1982 May;118(5):322-5. DOI: 10.1001/archderm.118.5.322

8. Narang T, Dogra S, Kaur I, Kanwar AJ. Malassezia and psoriasis: Koebner's phenomenon or direct causation? J Eur Acad Dermatol Venereol. 2007 Sep;21(8):1111-2. DOI: $10.1111 / j .1468-3083.2006 .02097 . x$

9. Bunse T, Mahrle G. Soluble Pityrosporum-derived chemoattractant for polymorphonuclear leukocytes of psoriatic patients. Acta Derm Venereol. 1996 Jan;76(1):102.

10. Gaitanis G, Magiatis P, Hantschke M, Bassukas ID, Velegraki A. The Malassezia genus in skin and systemic diseases. Clin Microbiol Rev. 2012 Jan;25(1):10641. DOI: 10.1128/CMR.00021-11

11. Ashbee HR, Evans EGV. Immunology of diseases associated with Malassezia species. Clin Microbiol Rev. 2002 Jan;15:21-57. DOI: 10.1128/CMR.15.1.2157.2002

12. Ertam I, Aytimur D. Malassezia spp. ve Dermatoloji'deki Yeri. Turkderm. 2006;40(1):7-10.

13. Gupta AK, Batra R, Bluhm R, Boekhout T, Dawson TL. Jr. Skin diseases associated with Malassezia species. J Am Acad Dermatol. 2004 Nov;51(5):785-98. DOI: 10.1016/j.jaad.2003.12.034

14. Kantarcioglu AS, Yucel A. Malassezia türleri: taksonomi, mikoloji, immünoloji, patogenez, vücuttaki dağılımı ve ilişkili infeksiyonlar, laboratuvar tanımı, antifungallere duyarlılığı. Cerrahpaşa J Med. 2005;36:134-54.

15. Rodoplu G, Saracli MA, Gumral R, Yildiran ST. Distribution of Malassezia species in patients with pityriasis versicolor in Turkey. Journal de Mycologie Medicale. 2014 June;24(2):117-23. DOI: 10.1016/j.mycmed.2014.02.001

16. Midgley G, Gueho E, Guillot J. Disease caused by Malassezia species. Topley and Wilson's Microbiology and microbial infections. 9th edition. Edited by: Ajello L, Hay RJ. London: Arnold 1998;4:201-11.

17. Bayraktar S, Duran N, Duran GG, Eryilmaz N, Aslan $\mathrm{H}$, Onlen $\mathrm{C}$, et al. Identification of medically important Candida species by polymerase chain reaction-restriction fragment length polymorphism analysis of the rDNA ITS1 and ITS2 regions. Indian J Med Microbiol. 2017 Oct-Dec;35(4):535-42. DOI: 10.4103/ijmm. IJMM_17_102

18. Thomas $\overline{\mathrm{DS}}$, Ingham E, Bojar RA, Holland KT. In vitro modulation of human keratinocyte pro- and anti-inflammatory cytokine production by the capsule of Malassezia species. FEMS Immunol Med Microbiol. 2008 Nov;54(2):203-14. DOI: 10.1111/j.1574695X.2008.00468.x

19. Kesavan S, Holland KT, Ingham E. The effects of lipid extraction on the immunomodulatory activity of Malassezia species in vitro. Med Mycol. 2000 Jun;38(3):23947. DOI: $10.1080 / \mathrm{mmy} .38 .3 .239 .247$

20. Belew PW, Rosenberg EW, Jennings BR. Activation of the alternative pathway of complement by Malassezia ovalis (Pityrosporum ovale). Mycopathologia. 1980;70(3):187-91. DOI: 10.1007/BF00443030

21. Squiquera L, Galimberti R, Morelli L, Plotkin L, Milicich R, Kowalckzuk A, et al. Antibodies to proteins from Pityrosporum ovale in the sera from patients with psoriasis. Clin Exp Dermatol. 1994 Jul;19(4):289-93. DOI: 10.1111/j.1365-2230.1994.tb01197.x

22. Aydogan K, Tore O, Akcaglar S, Oral B, Ener B, Tunalı $\mathrm{S}$, et al. Effects of Malassezia yeasts on serum Th1 and Th2 cytokines in patients with guttate psoriasis. Int J Dermatol. 2013 Jan;52(1):46-52. DOI: 10.1111/j.13654632.2011.05280.x

23. Baroni A, Paolett I, Ruocc E, Agozzin M, Tufan MA, Donnarumma G. Possible role of Malassezia furfur in psoriasis: modulation of TGF- $\beta 1$, integrin, and HSP70 expression in human keratinocytes and in the skin of psoriasis-affected patients. J Cutan Pathol. 2004 Jan;31(1):35-42. DOI: 10.1046/j.03036987.2004.0135.x

24. Baroni A, Orlando M, Donnarumma G, Farro P, Iovene MR, Tufano MA, et al. Toll-like receptor 2 (TLR2) mediates intracellular signalling in human keratinocytes in response to Malassezia furfur. Arch Dermatol Res. 2006 Jan;297(7):280-8. DOI: 10.1007/s00403-0050594-4

25. Plotkin LI, Mathov I, Squiquera L, Leoni J. Arachidonic acid released from epithelial cells by Malassezia furfur phospholipase A2: a potential pathophysiologic mechanism. Mycologia. 1998;90:163-9. DOI: 10.1080/00275514.1998.12026895

26. Brunke S, Hube B. MfLIP1, a gene encoding an extracellular lipase of the lipid-dependent fungus Malassezia furfur. Microbiology. 2006 Feb;152:547-54. DOI: 10.1099/mic. 0.28501-0

27. Oh BH, Song YC, Lee YW, Choe YB, Ahn KJ. Comparison of Nested PCR and RFLP for Identification and Classification of Malassezia Yeasts from Healthy $\mathrm{Hu}-$ man Skin. Ann Dermatol. 2009 Nov;21(4):352-7. DOI: 10.5021/ad.2009.21.4.352

28. Prohic A. Identification of Malassezia species isolated from scalp skin of patients with psoriasis and healty subjects. Acta Dermatovenerol Croat. 2003;11(1):10-6.

29. Takahata Y, Sugita T, Hiruma M, Muto M. Quantitative analysis of Malassezia in the scale of patients with 
psoriasis using a real-time polymerase chain reaction assay. Br J Dermatol. 2007 Oct;157(4):670-3. DOI: 10.1111/j.1365-2133.2007.08090.x

30. Paulino L, Tseng C, Blaser MJ. Analysis of Malassezia microbiota in healthy superficial human skin and psoriatic lesions by multiplex real-time PCR. FEMS Yeast Res. 2008;8:460-71. DOI: 10.1111/j.15671364.2008.00359.x

31. Gomez-Moyano E, Crespo-Erchiga V, Martínez-Pilar L, Godoy Diaz D, Martínez- Garcia S, Lova Navarro M, et al. Do Malassezia species play a role in exacerbation of scalp psoriasis? J Mycol Med. 2014 Jun;24(2):87-92. DOI: $10.1016 /$ j.mycmed.2013.10.007

32. Tarazooie B, Kordbacheh P, Zaini F, Zomorodian K, Saadat F, Zeraati H, et al. Study of the distribution of Malassezia species in patients with pityriasis versicolor and healty individuals in Tehran, Iran. BMC Dermatol. 2004 May;4:5. DOI: 10.1186/1471-5945-4-5

33. Gupta AK, Kohli Y, Summerball RC, Faergemann J. Quantitative culture of Malassezia species from different body sites of individuals with or without dermatoses. Med Mycol. 2001 Jun;39(3):243-51. DOI: 10.1080/714031025

34. Jagielski T, Rup E, Ziółkowska A, Roeske K, Macura $\mathrm{AB}$, Bielecki J. Distribution of Malassezia species on the skin of patients with atopic dermatitis, psoria- sis, and healthy volunteers assessed by conventional and molecular identification methods. BMC Dermatol. 2014 Mar;14:3. DOI: 10.1186/1471-5945-14-3

35. Tett A, Pasolli E, Farina S, Truong DT, Ascinar F, Zolfo $\mathrm{M}$, et al. Unexplored diversity and strain-level structure of the skin microbiome associated with psoriasis. NPJ Biofilms Microbiomes. 2017 Jun;3:14. DOI: 10.1038/ s41522-017-0022-5

36. Amaya M, Tajima M, Okubo Y, Sugita T, Nishikawa A, Tsuboi R. Molecular analysis of Malassezia microflora in the lesional skin of psoriasis patients. J Dermatol. 2007 Sep;34(9):619-24. DOI: 10.1111/j.13468138.2007.00343.x

37. Stehlikova Z, Kostovcik M, Kostovcikova K, Kverka M, Juzlova K, Rob F, et al. Dysbiosis of Skin Microbiota in Psoriatic Patients: Co-occurrence of Fungal and Bacterial Communities. Front Microbiol. 2019 Mar;10:438. DOI: 10.3389/fmicb.2019.00438

38. Rudramurthy SM, Honnavar P, Chakrabarti A, Dogra S, Singh P, Handa S. Association of Malassezia species with psoriatic lesions. Mycoses. 2014 Aug;57(8):483-8. DOI: $10.1111 /$ myc. 12186

39. Prohic A, Jovovic Sadikovic T, Krupalija-Fazlic M, Kuskunovic-Vlahovljak S. Malassezia species in healthy skin and in dermatological conditions. Int J Dermatol. 2016 May;55(5):494-504. DOI: 10.1111/ijd.13116 